\title{
BARS IN CUSPS
}

\author{
WALTER DEHNEN \\ Theoretical Physics \\ 1 Keble Road, Oxford OX1 3NP, UK
}

In order to investigate the stability properties of galaxy models with central density cusps, $N$-body simulations of oblate models with density $\rho \propto m^{-1}$ $(m+a)^{-3}$ where $m^{2}=R^{2}+[z / q]^{2}$ and distribution functions $f\left(E, L_{z}\right)$ (computed as in Dehnen, 1995) have been performed with the following results.

1. An E7 model with identical amounts of stars of either sense of rotation was stable over $30 t_{\mathrm{dyn}}(r=a)$. This is interesting for the bending instability has been argued to set in at about this flattening and be responsible for the absence of flatter elliptical galaxies (Merritt \& Sellwood, 1994).

2. Rapidly rotating $\mathrm{E} \gtrsim \mathrm{E} 5$ models quickly form weak bars inside the cusp, which are stronger for the more flattened, faster rotating initial configurations. The bars grow in a self similar fashion from inside out: the pattern speed decreases with increasing bar length and time. This process is initiated at the origin, where, because of finite $N$, the actual density no

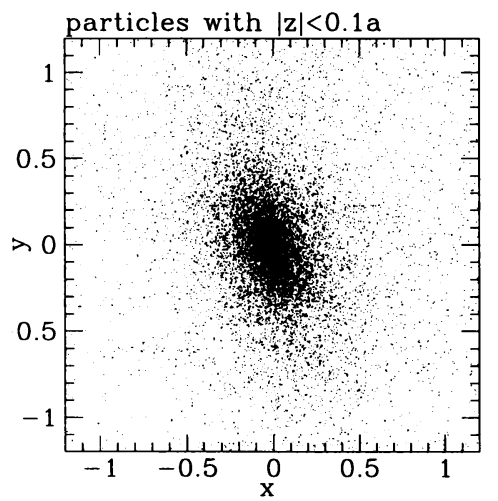
longer follows the power law, and stops when the edge of the cusp is reached. A typical example is given in the figure showing the $x$ - $y$-coordinates of particles with $|z|<0.1 a$ after $\sim 20 t_{\mathrm{dyn}}(r=a)$ for an initially rapidly rotating E7-model. The bar has axis ratios of about 5:3:1, and extends almost to corotation. However, it has no sharp edge, but an inhomogenous density with a cusp steeper than the initial model. No sign of a buckling instability has been observerd.

\section{References}

Dehnen W., 1995, MNRAS, 274, 919

Merritt D., Sellwood J., 1994, ApJ, 425, 551 